\title{
Keyless Smart Home: An Application of Home Security and Automation
}

\author{
AMIRITA DEWANI ${ }^{1 *}$, SANIA BHATTI ${ }^{1}$, PIRAH MEMON ${ }^{1}$, \\ VEENA KUMARI $^{2}$, ANUM ARAIN ${ }^{1}$ and AYAZ JISKANI ${ }^{1}$
}

\begin{abstract}
${ }^{1}$ Department of Software Engineering, MUET, Jamshoro, Pakistan.
${ }^{2}$ Institute of Information Technology, University of Sindh Hyderabad, Pakistan.
\end{abstract}

\begin{abstract}
The rapid and revolutionary advancement in technology has increased the demand for advanced ubiquitous mobile applications in our daily lives. Home automation system uses collective smart technology to elevate sphere of security and human living standards. Wireless Smart home systems have been prototyped by researchers to realize different applications in vast contexts. In this paper we propose a keyless smart home security system which uses Bluetooth as a communication protocol to automate door locks based on a security code and predefined user credentials. The system combines Android phone as a signal sender to dispatch the input signal from an end users side, Bluetooth communication module as signal receiver, ARDUINO microcontroller as CPU and servo motor and light emitting diodes as an output. The system integrates two modules: one of which is Arduino microcontroller which is responsible for authentication and verification of security code and allows servo motor to process the output according to the received input. The second is android Application which provides an interface to the user to set credentials and security code which is then transmitted to the Arduino microcontroller via Bluetooth technology. Since Arduino and Android are open source, hence implementation rate of the proposed system is inexpensive and feasible.
\end{abstract}

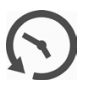

Article History

Received: 10 April 2018

Accepted: 06 May 2018

\section{Keywords}

Home security, Home automation, Keyless home Door,

Android,

Bluetooth

\section{Introduction}

Due to encroachment of smart phones in terms of powerful processors, larger storage, affluent entertainment functions and variety of communication techniques their users are amplifying swiftly. According to the Gartner Research, in the market of smartphone operating system, the Android has captured $81.7 \%$ (352 million) of the total market in the fourth quarter of 2016 and also grew its market share by $3.2 \%$, iOS captured $17.9 \%$ (77 million), Windows captured 0.3 , Blackberry captured $0.0 \%$, and other OS captured $0.1 \%{ }^{1}$. A Smart Home system

CONTACT Amirita Dewani (C) 2018 The Author(s). Published by Techno Research Publishers

This is an 6 Open Access article licensed under a Creative Commons Attribution-NonCommercial-ShareAlike 4.0 International License (https://creativecommons.org/licenses/by-nc-sa/4.0/), which permits unrestricted NonCommercial use, distribution, and reproduction in any medium, provided the original work is properly cited.

To link to this article: http://dx.doi.org/10.13005/ojcst11.02.06 
has numerous advantages and applications; for example it can open or close the front door for known visitors, may adjust the light according to the mood of the residential of home, may be used for turning on/ off the electronic things and many more. The trade organization CEDIA (Custom Electronic design and Installation Association) has termed Smart Home as multibillion-dollar business. According to the prediction of Research firm Markets and Markets, the Smart Home market will be US $\$ 120$ billion industry by $2022^{2}$. Almost every smartphone could be used to develop Smart Home products. Smart phones are now called pocketable PCs and are being used to develop smart home products using different technologies such as $\mathrm{Wi}-\mathrm{Fi}$, Infrared and Bluetooth technology.

The Bluetooth is the most trusted name in wireless connection. It is a global wireless standard for providing the secure connectivity. Whether it is about building automation, improving medical devices or implementing smart home/city, Bluetooth technology always opens door for achieving such goals. Bluetooth is a solution to those tasks which require physical wires connections. For instance, the Bluetooth enabled printer, wirelessly, laptop, desktop or mobile devices can be connected. It requires low-cost transceiver chip included in device. The transceiver operates under the $2.45 \mathrm{GHz}$ frequency band. Bluetooth can link digital devices between range of 10 meters to 100 meters and the data can be exchanged at the rate of $1 \mathrm{Mbps}$ up to $3 \mathrm{Mbps}$ depending on the Bluetooth device class ${ }^{3}$.

Security is the primary requirement of every user. Password based authenticated key exchange is a protocol that is used to provide strong authentication 4 . Keeping keys and opening the door with hands is headache. Technology brought keyboard based door lock systems where user can type his password and get access to it, but this technology compels user to come on the door and type the password. So, with the attributes of Bluetooth technology and password, we offer a cost efficient system which enables user to access the door wirelessly from a particular range using their smart phones by inputting a unique key. A combination of Android Phone and Arduino Microcontroller can make it possible to assemble a door lock system which can be locked and unlocked remotely from a specific range.

\section{Literature Review}

A variety of door automation systems have been proposed in literature which are controllable via Bluetooth $^{9-11}, \mathrm{RFID}^{5}$, fingerprint ${ }^{6-8}, \mathrm{WiFi}^{12}$ etc. Some of the recent works are highlighted in this section.

The RFID technology can be used for many purposes such as tracking of people, tracking of inventory, control of applications, security etc. Authors in a study ${ }^{5}$ implemented a system for opening and closing of the door using RFID technology to validate the user to unlock the door. This process involved the contact of users' tags to the RFID reader. The developed system is also able to maintain a log containing check-in and check-out of each user along with basic information of the users. Wong et al., in their final year project at The Hong Kong University of Science and Technology developed a home security system using fingerprint as the method of authentication ${ }^{6}$.

The system is connected to a mobile application over the Internet which is allowing users to monitor entrants through camera surveillance and had remote access to the door. This project aimed for improving home security and convenience in accessing doors. The limitation in their work is that the Android smartphone must support fingerprint sensor and internet access, otherwise the door will not be in a position to be accessed. Other finger print based door locking systems are proposed $\mathrm{in}^{7,8}$. The proposed systems ${ }^{7,8}$ are based on existing biometrics systems and stored fingerprints of authorized users. The systems authorize access after confirming matching of collected fingerprints with already stored biometrics in the database. Android based control system providing the safety of home's main door and car door are presented in ${ }^{9,10}$. The systems become accessible after establishing Bluetooth connections and validate the user login details such as username and password. In addition the systems are also able to control the appliances of a room. The limitation of their work is that they are not providing the option to rest the password. This limitation is addressed by Ankit et al., ${ }^{11}$ and the user is allowed to change the current password. In this work Arduino UNO microcontroller and password are used to control the door and appliances of a room. 
EIShafee and Alaa Hamed ${ }^{12}$ have presented WiFi based control system to maintain the temperature, detect smoke, door status and light level. The system consists of web interface module for monitoring and controlling purposes. The hardware module consists of four assorted PCBS and is used to provide interfaces for sensors and actuators. The system in ${ }^{18}$ uses ZigBee module in digital door lock and the door locks act as a central main controller of the overall home automation system. The developed system is based on network of sensors nodes, actuators with digital door lock as a base system. Hence proposed system is limited to sensors, RFID and ZigBang model.

N. Sriskanthan ${ }^{19}$ explained the model for home automation using Bluetooth via PC. But unfortunately the system lacks to support mobile technology. Deepal in ${ }^{20}$ explains proposed system based on ADK (Accessory Development kit) at home. The system is based on automation for monitoring and controlling home appliances via android mobile phone or tablet.

Since Bluetooth wireless technology is low cost and has become pervasive in smart phones, therefore this research work has proposed and realized a concept of keyless smart home using android platform and Bluetooth as a communication protocol. The major aim is to provide automated door access and security management in a cost efficient manner.

\section{Hardware Architecture \& Software \\ Specifications \\ Hardware Architecture}

The hardware section of proposed approach comprises of the following components.

- ARDUINO-UNO

- Servo Motor

- $\quad$ Android based Smart Phone

- $\quad$ Bluetooth Module (HC-05)

- $\quad$ Display unit (LEDs)

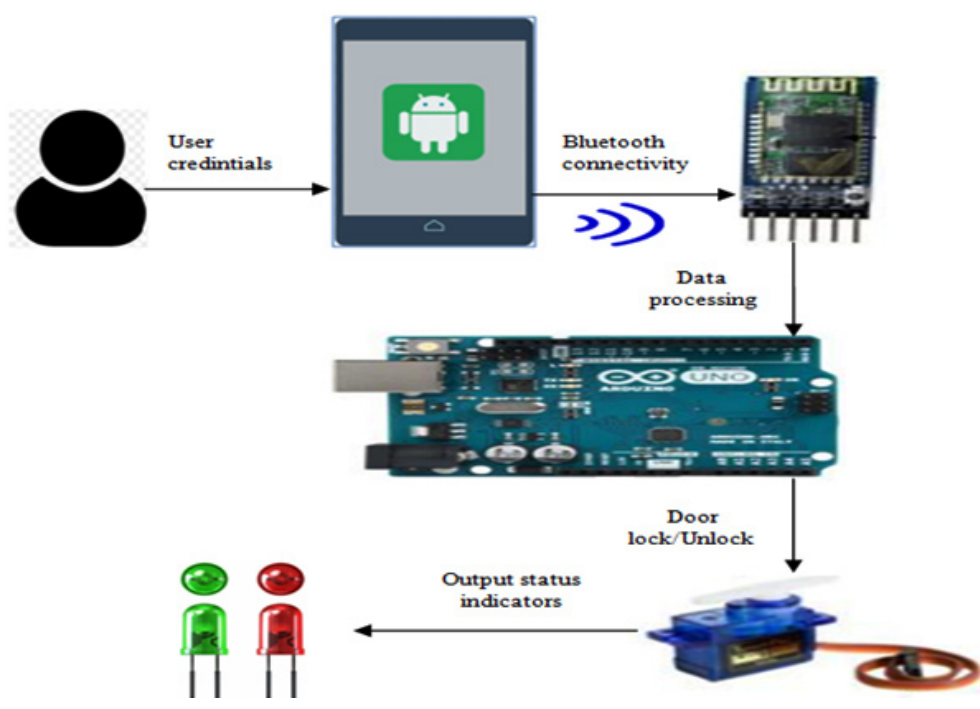

Fig. 1: System Hardware-Block Diagram

\section{Arduino-UNO}

Arduino a small microcontroller board having various connection sockets to be coupled with different electronic devices like motors, sensors relays etc. It can be programmed to accomplish desired tasks independently or can be controlled via a computer. It can either be powered by a $12 \mathrm{v}$ battery or a USB plug connected to a computer ${ }^{11}$. In the proposed research work Arduino-UNO microcontroller acts as a processing unit and is programmed to perform various logical functions. It also interfaces with other hardware components.

\section{Android Smart Phone}

The smart phone communicates with the micro controller by making connection with the Bluetooth 
module (HC-05). It sends the code to the smart phone through which the door gets locked/unlocked. The smart phone gets the security credentials from the user as an input. If it is verified that the user is authorized and authenticated, the smart phone will send the code to the microcontroller which further instructs the servo motor to access (lock/unlock) the door. The front end module (Android App) gets connected with the hardware module via the MAC address of Bluetooth module HC-05.

\section{Bluetooth Module (HC-05)}

Bluetooth Module works as a bridge between smart phone and microcontroller ${ }^{13}$. The Bluetooth Module consists of 6 pins out of which 4 pins are used to communicate and perform required function. The VCC is connected to $5 \mathrm{~V}$ pin, ground is connected to gnd pin, TX pin of is connected to TX(1) pin of Arduino-UNO which is used to send data from Arduino-UNO to Android Phone and the RX pin of module $R X(0)$ is used to receive and send the data from Android phone to Aurduino-UNO.

\section{LEDs}

LEDs are used to indicate status of the system output. The green light shows that the door is open where as the red light shows the door is locked. The Green LED is connected to the pin 10 and gnd where as the Red LED is connected to the pin 11 and gnd. Both LEDs are connected to same gnd to reduce wiring expenses.

\section{Servo Motor}

A servomotor behaves as a rotating/linear actuator and controls acceleration, velocity and angular or linear position of the object in a precise manner. Servo motor is a DC motor coupled with a position sensor which rotates at its axis from 0 to 180 degrees. This motor can be programmed for several purposes. The Servo motor has three pins. The first one is signal pin which is connected to the pin 3 and is programmed to send signal to the motor. The second pin is VCC pin which is connected to $3.3 \mathrm{~V}$ pin of Arduino-UNO and the third pin is ground pin which is connected to gnd pin of Arduino-UNO.

The hardware modules and the role they play in proposed keyless smart home are summarized in the table-1.

Table 1: Functionality of System Components

\begin{tabular}{|c|c|c|}
\hline $\begin{array}{l}\text { S. } \\
\text { No }\end{array}$ & System Component & Role/Functionality \\
\hline 1. & Android Smart phone & Acts as a user front end \\
\hline 2. & Bluetooth Module(HC-05) & Act as a connectivity module to transmit data \\
\hline 3. & Servo Motor & Acts as a rotator to control door lock \\
\hline 4. & Display unit (LEDs) & Acts as an output indicators \\
\hline 5. & Arduino-UNO & Acts as a CPU for data processing \\
\hline
\end{tabular}

\section{Software Specifications Android Platform}

The smart mobile terminal platform Android is getting popular all over the world with its wide variety of appliances and enormous use in numerous spheres of our daily life. We all know that we are in $21^{\text {st }}$ century and we know that technology have improved a lot from the past century. Android platform is composed of operating system, user interface and application components which allow developer freedom access and modify the source code. So Android is providing a free platform to the developers with numerous facilities to generate new applications in a rapid rate ${ }^{14}$. Since, all Bluetooth applications work coherently with every version of android, our application will apparently run on any version of android.

\section{Eclipse IDE}

Front end user App is developed using Eclipse IDE and Java programming language. Eclipse is an integrated development environment (IDE) used in computer programming, and is the most widely used Java IDE. It contains a base workspace and 
an extensible plug-in system for customizing the environment ${ }^{15}$.

\section{SQLite Library}

SQLite is the most widely deployed in-process library that implements a SQL database engine. It offers high storage efficiency, fast query operation and small memory needs. Due to the fact that a complete SQLite database is stored in a single cross-platform disk file and SQLite does not support multiple users, anyone who has direct access to the file can read the whole database content ${ }^{16}$.

\section{Arduino IDE}

The Arduino Integrated Development Environment or Arduino Software (IDE) contains a text editor for writing code, a message area, a text console, a toolbar with buttons for common functions and a series of menus. It connects to the Arduino and Genuino hardware to upload programs and communicate with them ${ }^{17}$.

\section{System Workflow Mechanism}

The overall system workflow is shown in fig-2.

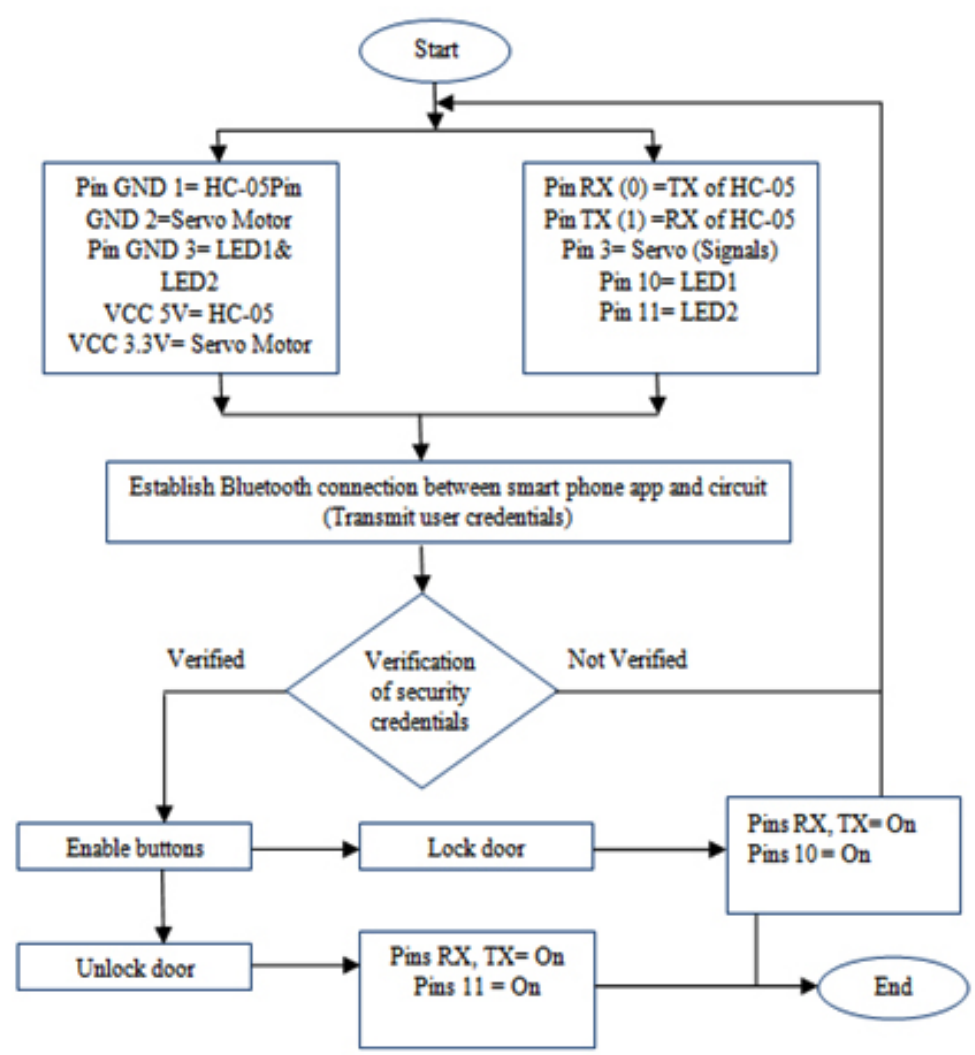

Fig. 2: System Workflow mechanism

There are various connections and pins in the systems which are used to perform specific tasks. Mainly these pins/connections fall into two categories. The first one is power (VCC and Ground) and other is related to signal management and transmission. There are three ground pins in the ARDUINO-UNO. The first GND pin is connected to the GND pin of $\mathrm{HC}-05$ Bluetooth module, the second one is connected to the GND pin of Servo Motor and the third GND pin is used for GND of both LEDs (used as an indicator/ output module). The $\mathrm{HC}-05$ Bluetooth module requires $5 \mathrm{~V}$ at its VCC pin; therefore it is connected to the VCC $5 \mathrm{~V}$ pin of ARDUINO-UNO module. Servo motor requires 3.3V at it VCC pin; therefore it is connected to the VCC 3.3V of ARDUINO-UNO. Observing the signal 
section, it can be seen that the pins of devices are connected to their respective pins of ARDUINO-UNO as they are programmed.

User access the system via front-end android app. Front end user App is developed using Eclipse software and Java programming language. It is immensely important to establish connection via Bluetooth protocol before security credentials can be transmitted. Once the connection is established and security credentials are verified, the control buttons to remotely lock/unlock a door gets enabled. When the end-user presses lock/unlock button the smart phone sends command to the circuit which is used to lock/unlock the door as they are programmed. At circuit side, Pin TX of $\mathrm{HC}-05$ is connected to the $\mathrm{RX}(0)$ of ARDUINO-UNO board to receive data from the smart phone where as pin $\mathrm{RX}$ of $\mathrm{HC}-05$ is connected to the TX(1) of the ARDUINO-UNO board to send the data to the smart phone. When user sends command to circuit from the smart phone. The circuit matches the command and sends current to the pin 3, where the signal pin of Servo motor is connected and it rotates the Servo motor from 00 to 1800 to lock/unlock the door. During this circuit is also programmed to light Red LED for locked door and Green LED for unlocked door as indicators by sending current to the pin 10 and pin 11 of ARDUINO-UNO board, where both LEDs are connected.

The implementation of proposed system is depicted in figure-3.

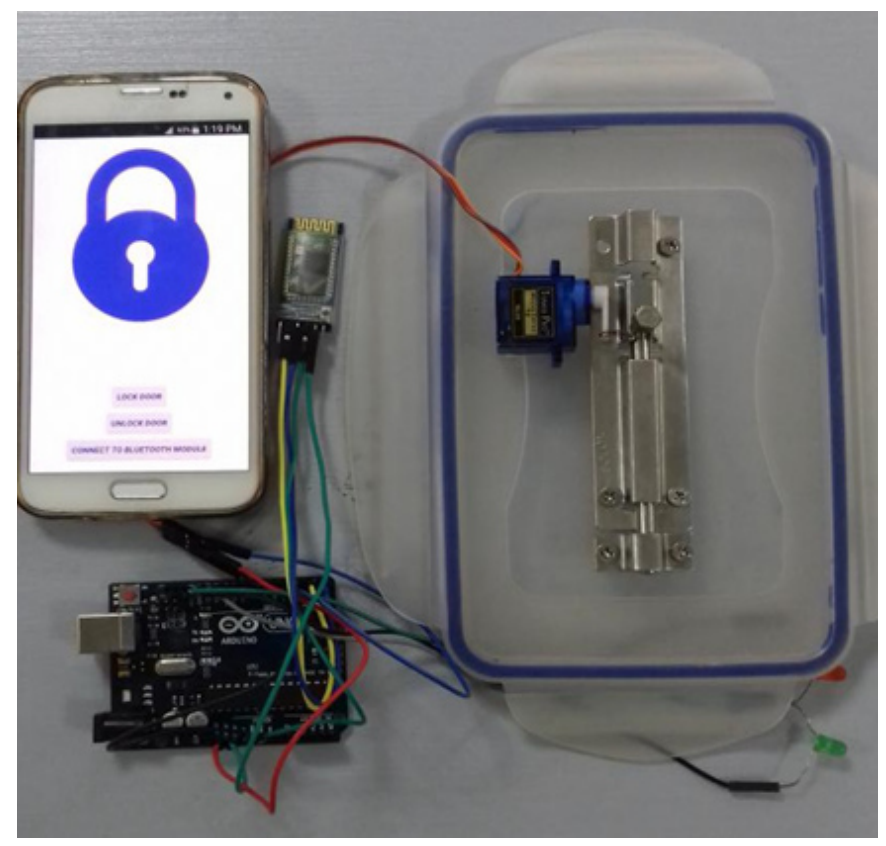

Fig. 3: System implementation

The Arduino-UNO Microcontroller is used to perform logical tasks and functions. The ArduinoUNO Microcontroller receives the data from Android phone. For communication with Arduino using Bluetooth, a Bluetooth module needs to be connected with Arduino. This connection is established via Bluetooth Module (HC-05). HC-05 module has 6 pins. We have connected 4 pins to Arduino, they are: RXD TXD VCC GND. RXD will receive data from Arduino; TXD will send data to
Arduino; $\mathrm{VCC}$ is the power supply (3.3V to $6.6 \mathrm{~V}$ ) and GND is the ground. On the other hand the Servo Motor plays a vital role. The Servo motor has three pins. The first one is signal pin which is connected to the pin which is programmed to send signal to the motor, the VCC pin is connected to $3.3 \mathrm{~V}$ pin or Arduino-UNO and the ground pin is connected to gnd pin of Arduino-Board. LEDs are used to indicate. The green light shows that the door is open where the red light shows that door is locked. 


\section{Conclusion and Dicussion}

Present era has witnessed Home automation and related applications as a growing trend. It is a technology that remoulds the manual work to the automation in order to enhance luxury, comfort and standard of living thereby making human life much easier, better and more secure. This paper presents an idea of keyless smart home and an application of home automation. The proposed approach resolves problems presented by conventional metal locks of existing doors i-e vulnerability to break in's, the space carried by the lock and human efforts required to open/close the door manually. Moreover the implementation rate of developed system is very low there by making it affordable for a common person. As compared to most of the existing systems, as discussed in literature review, the keyless smart home is easier to install and maintain. It supports mobile technology and open source android system platform. The proposed approach is realized using Bluetooth technology for connectivity, android based smart phone App for end user access and user credentials for security and authentication. ARDUINO microcontroller, Bluetooth module, servo motor, male and female wires and front-end android app were interfaced and integrated to realize the desired architecture. In future the designed system can be extended to embrace other aspects of home automation and security. Furthermore this research work can be enhanced to support other OS platforms apart from android.

\section{Acknowledgment}

We are extremely grateful to department of software engineering, Mehran University of Engineering and Technology, Jamshoro for all the technical support to accomplish this project.

\section{References}

Gartner homepage, available, https://www. gartner.com/technology/home.jsp The institute blog [online], available http:// theinstitute.ieee.org/ieee-roundup/blogs/blog/ is-it-smart-to-have-a-smart-home

3 Collotta, Mario, et al., "Bluetooth 5: a concrete step forward towards the loT." arXiv preprint arXiv:1711.00257 (2017).

4 Chang, Ting-Yi, Min-Shiang Hwang, and Chou-Chen Yang. "Password Authenticated Key Exchange and Protected Password Change Protocols." Symmetry 9.8 (2017): 134.

5 Verma, Gyanendra K., and Pawan Tripathi. "A digital security system with door lock system using RFID technology." International Journal of Computer Applications (IJCA)(0975-8887) 5.11 (2010).

$6 \quad$ Kartika Wong, Felix Hoi Chak Satya, Uriel Jethro "Smartphone-Controlled FingerprintEnabled Door Lock System.", Department of Electronic and Computer Engineering The Hong Kong University of Science and Technology, Final Year Project Report, April 15, 2015.

7 Shankar, A. Aditya, et al. "Finger Print Based
Door Locking System." International Journal of Engineering and Computer Science 4.3 (2015): 10810-10814.

8 Anu, and Dinesh Bhatia. "A smart door access system using finger print biometric system." International Journal of Medical Engineering and Informatics 26.3 (2014): 274-280.

9 Kamelia, Lia, et al.,"DoorAutomation System Using Bluetooth-BasedAndroid For Mobile Phone." ARPN Journal of Engineering and Applied Sciences 9.10 (2014): 1759-1762.

10 Sadeque Reza Khan, Farzana Sultana Dristy, "Android based security and home automation system", International Journal of Ambient Systems and Applications (IJASA), Vol. 3, No. 1, pp. 15-24, March 2015.

11 Jain, Ankit, Anita Shukla, and Ritu Rajan. "Password Protected Home Automation System with Automatic Door Lock.", MIT International Journal of Electrical and Instrumentation Engineering, Vol.6, No. 1, January 2016, pp. 28-31.

12 EIShafee, Ahmed, and Karim Alaa Hamed. "Design and implementation of a WIFI based home automation system." World academy of science, engineering and technology 68 
(2012): 2177-2180.

13 Kamelia, Lia, et al., "DoorAutomation System Using Bluetooth-BasedAndroid For Mobile Phone." ARPN Journal of Engineering and Applied Sciences 9.10 (2014): 1759-1762.

14 Khan, Sadeque Reza, and Farzana Sultana Dristy. "Android based security and home automation system." arXiv preprint arXiv:1504.03564 (2015).

15 Eclipse [online], available at: https://eclipse. org/ide/

16 Mutti, Simone, Enrico Bacis, and Stefano Paraboschi. "Sesqlite: Security enhanced sqlite: Mandatory access control for android databases." Proceedings of the $31^{\text {st }}$ Annual Computer Security Applications Conference. ACM, 2015.
17 Arduino Software (IDE) [online], available at: https://www.arduino.cc/en/Guide/ Environment.

18 Y. T. Park, P. Sthapit, and J.-Y. Pyun, "Smart digital door lock for the home automation," in TENCON 2009-2009 IEEE Region 10 Conference, 2009, pp. 1-6: IEEE.

19 N. Sriskanthan and Tan Karand. "Bluetooth Based Home Automation System". Journal of Microprocessors and Microsystems, Vol. 26, pp.281-289, 2002.

20 D. Javale, M. Mohsin, S. Nandanwar, and M. Shingate, "Home automation and security system using Android ADK," International journal of electronics communication and computer technology (IJECCT), vol. 3, no. 2, pp. 382-385, 2013. 